\title{
Evaluation des Risikoorientierten Sanktionenvollzugs - Konstruktion einer Veränderungsmessung
}

\author{
Daniel Treuthardt ${ }^{1,2} \cdot$ Melanie Kröger ${ }^{3}$ \\ Eingegangen: 23. Oktober 2020 / Angenommen: 27. November 2020 / Online publiziert: 7. Januar 2021 \\ (c) Der/die Autor(en) 2021
}

\section{Zusammenfassung}

Der Risikoorientierte Sanktionenvollzug (ROS) hat zum Ziel, Rückfälle größtmöglich zu reduzieren und Ressourcen zu stärken. In verschiedenen Evaluationen wurde ROS untersucht und weiterentwickelt. Nun soll eine Veränderungsmessung konzipiert werden, die ROS erweitert und die Abbildung von Veränderungen transparenter und nachvollziehbarer machen soll. Hierzu werden sowohl personen- als auch umweltbezogene Gesichtspunkte herangezogen. Folgende 3 personenbezogenen Aspekte werden unterschieden: Veränderungspotenzial (welches sich aus Veränderungsfähigkeit und Veränderungsmotivation zusammensetzt), delinquenzbezogene Prozessvariablen und Stufen der Verhaltensänderung. Sowohl das Veränderungspotenzial als auch die delinquenzbezogenen Prozessvariablen erfassen die Bereiche Wollen, Wissen und Können. Die Stufen der Verhaltensänderung umfassen die Ebenen Absichtslosigkeit, Absichtsbildung, Handlung und Aufrechterhaltung. Die umweltbezogenen Aspekte umfassen zentrale Lebensbereiche wie Arbeit, Wohnen, soziales Umfeld etc. und werden anhand einer umweltbezogenen Veränderungsmessung bewertet, die ebenfalls die Bereiche Wollen, Wissen und Können umfasst. Umwelt- und personenbezogene Aspekte werden übergeordnet in der Ausprägung des Problemprofils betrachtet. Die Ausprägung des Problemprofils betrachtet, anders als die 3 personenbezogenen Aspekte, nicht nur konkrete deliktpräventive Aspekte, sondern auch die Auswirkung des Problemprofils in nichtdeliktrelevanten Situationen und Lebensbereichen. Abschließend werden Limitationen diskutiert sowie ein Ausblick auf eine zu einem späteren Zeitpunkt folgende Evaluation gegeben.

Schlüsselwörter Straftäterbehandlung · Veränderungsmessung · Personenbezogene Aspekte · Umweltbezogene Aspekte · Wirksamkeit

lic. phil. Daniel Treuthardt daniel.treuthardt@ji.zh.ch

1 Bewährungs- und Vollzugsdienste, ROS-Administration, Justizvollzug und Wiedereingliederung des Kantons Zürich, Hohlstrasse 552, 8090 Zürich, Schweiz
2 University of Zurich, Zürich, Schweiz

3 Fachbereich Psychologie, Universität Konstanz, 78457 Konstanz, Deutschland 


\title{
Evaluation of the risk-oriented enforcement of sanctions - construction of a change measurement
}

\begin{abstract}
The goal of the risk-oriented enforcement of sanctions (ROS) is to reduce the risk of reoffending as well as to strengthen offender's resources. Thus far, ROS has been evaluated and enhanced in several studies. Now a change measurement is constructed to further enhance the ROS. The aim is to make change in offenders more transparent and traceable. Therefore, environmental and personality aspects are considered. Personality aspects include: potential for change (consisting of ability to change and motivation to change), delinquency-related process variables and stages of change. Potential for change and delinquency-related process variables include the aspects of motivation, knowledge and ability. The stages of change comprise precontemplation, contemplation, action and maintenance. Environmental aspects (such as work life, financial situation and social environment) are measured with the environmental change measurement, which also includes motivation, knowledge and ability. Environmental and personality aspects are both considered to assess the overall manifestation of risk-related traits. While the other personality-based variables only consider delinquency-related aspects, the overall manifestation takes on a more holistic approach. Therefore, the influences of the risk-related patterns of thoughts and actions on everyday situations and environmental aspects are taken into account. Furthermore, limitations are discussed and an outlook is given on an evaluation that will take place in a different study.
\end{abstract}

Keywords Offender treatment $\cdot$ Change measurement $\cdot$ Personality aspects $\cdot$ Environmental factors $\cdot$ Effectiveness

\section{Einleitung}

Gemäß Andrews und Bonta (2010) haben jede Straftäterin und jeder Straftäter ein eigenes Risiko (,risk“), Bedürfnisse (,need“) und Ansprechvermögen (,,responsivity“). Dementsprechend sollte nach dem Risk-Need-Responsivity(RNR)Prinzip jede inhaftierte Person individuell zugeschnittene Unterstützung erfahren, um das Ziel des straffreien Lebens in sozialer Verantwortung zu erreichen.

Dem ersten Paragrafen des Strafvollzugsgesetz Nordrhein-Westfalen (StVollzG 2015) entsprechend, sei das Ziel der Freiheitsstrafe, Gefangene zu befähigen, künftig in sozialer Verantwortung ein Leben ohne Straftaten zu führen. Sinngemäß ist dies ebenfalls in den Strafvollzugsgesetzen der anderen Bundesländer zu lesen. Um dieses Ziel zu erreichen, sind in Deutschland psychosoziale Diagnoseverfahren verankert, auf deren Grundlage - in Übereinstimmung mit dem RNR-Prinzip - individuelle Vollzugspläne bzw. Vollzugs- und Eingliederungspläne oder Resozialisierungspläne erstellt werden.

In der Schweiz hingegen wurde als Reaktion auf unterschiedliche Rückfalltaten von Gewalt- und Sexualverbrechern in den Jahren 2010-2013 der Risikoorientierte Sanktionenvollzug (ROS) konzipiert und nach erfolgreicher Testung in den Kantonen der Deutschschweiz eingeführt (Treuthardt et al. 2019). Der ROS bezieht sich auf das RNRPrinzip und hat zum obersten Ziel, Rückfälle zu vermeiden sowie eine nachhaltige Resozialisierung der verurteilten Person zu fördern. Folgerichtig sind sowohl risikosenkende als auch ressourcenfördernde Interventionen elementar (Treuthardt et al. 2018).

\section{Fallverlauf im Risikoorientierten Sanktionenvollzug}

Jeder Fall durchläuft im ROS 4 Prozessschritte: Triage, $A b$ klärung, Planung und Verlauf (Abb. 1).

Im ersten Schritt, der Triage, wird anhand des Fall-Screening-Tools (FaST) der weitere Abklärungsbedarf festgestellt. Das FaST kategorisiert Fälle als A-, B- oder C-Fälle. A bedeutet hierbei, dass kein weiterer Abklärungsbedarf erkennbar ist; B, dass ein Fallresümee angezeigt ist, und $\mathrm{C}$, dass der Bedarf einer Risikoabklärung besteht. Das FaST kann und soll dementsprechend nicht feststellen, wie hoch das Rückfallrisiko und der Interventionsbedarf in dem entsprechenden Fall sind (für einen genaueren Überblick über das FaST: Treuthardt et al. 2018). Erst im nächsten Schritt, der Abklärung, werden anhand des Fallresümees oder der Risikoabklärung der Bedarf und das weitere Vorgehen zu Rückfallreduzierung und Ressourcenstärkung geplant. Hierfür müssen neben den forensischen Aspekten des personen- und umweltbezogenen Veränderungsbedarfs auch die juristischen Rahmenbedingungen und vollzugspraktischen Möglichkeiten berücksichtigt werden (Abb. 2).

Im weiteren Verlauf erfolgt dann eine regelmäßige standardisierte Berichterstattung durch die Arbeitspartner (Vollzugseinrichtung, Therapierende, Betreuende o. Ä.) an die fallverantwortliche Person der Vollzugsbehörde, die den Vollzug der Sanktion koordiniert und über die Gewährung von Vollzugsprogressionen entscheidet. Diese Berichte bilden die Grundlage für Vollzugsentscheidungen, beispielsweise über Lockerungen oder Vollzugsverschärfungen. 
Abb. 1 Prozessmodell des Risikoorientierten Sanktionenvollzugs

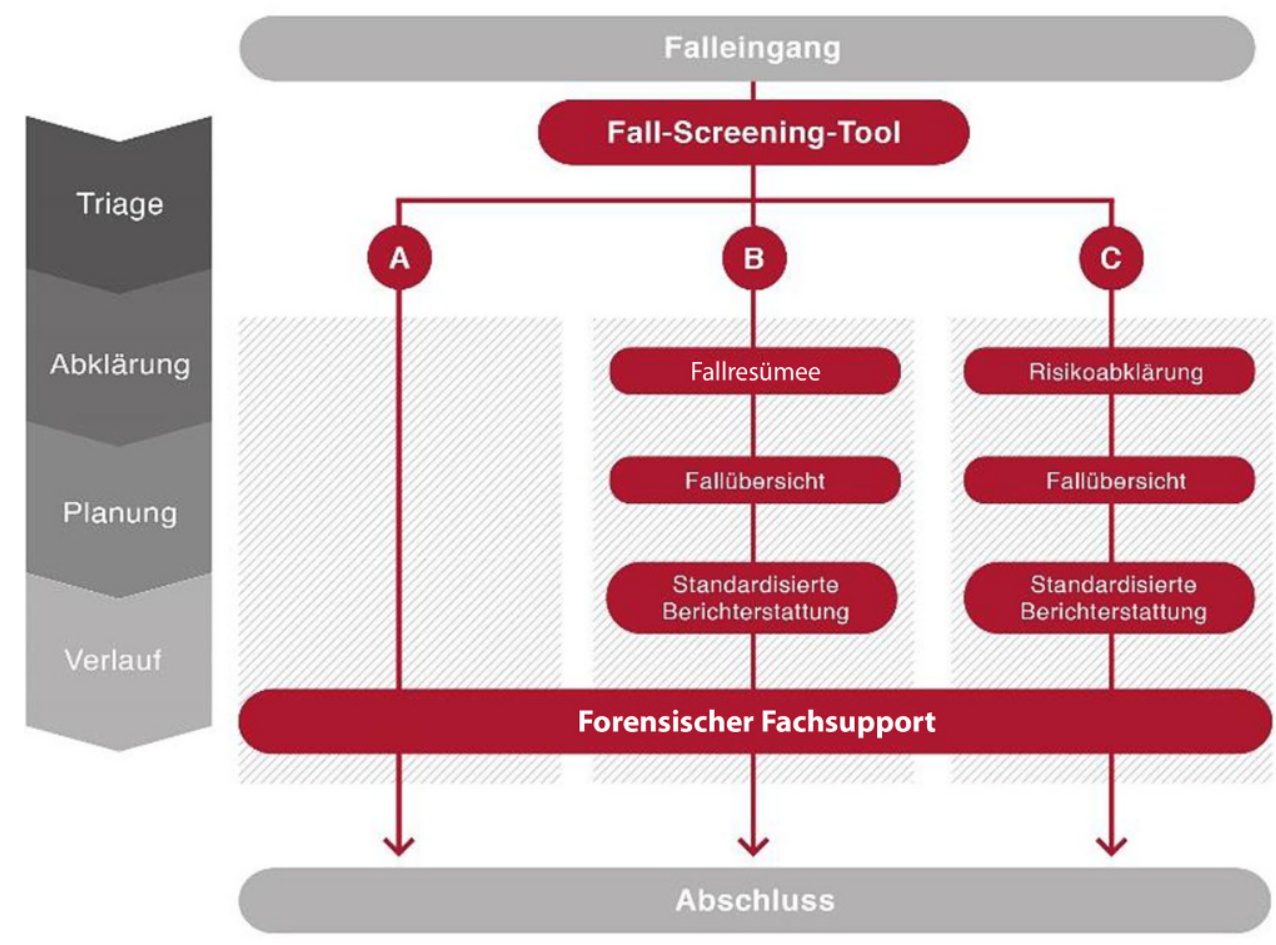

Abb. 2 Relevante Perspektiven im Justizvollzug

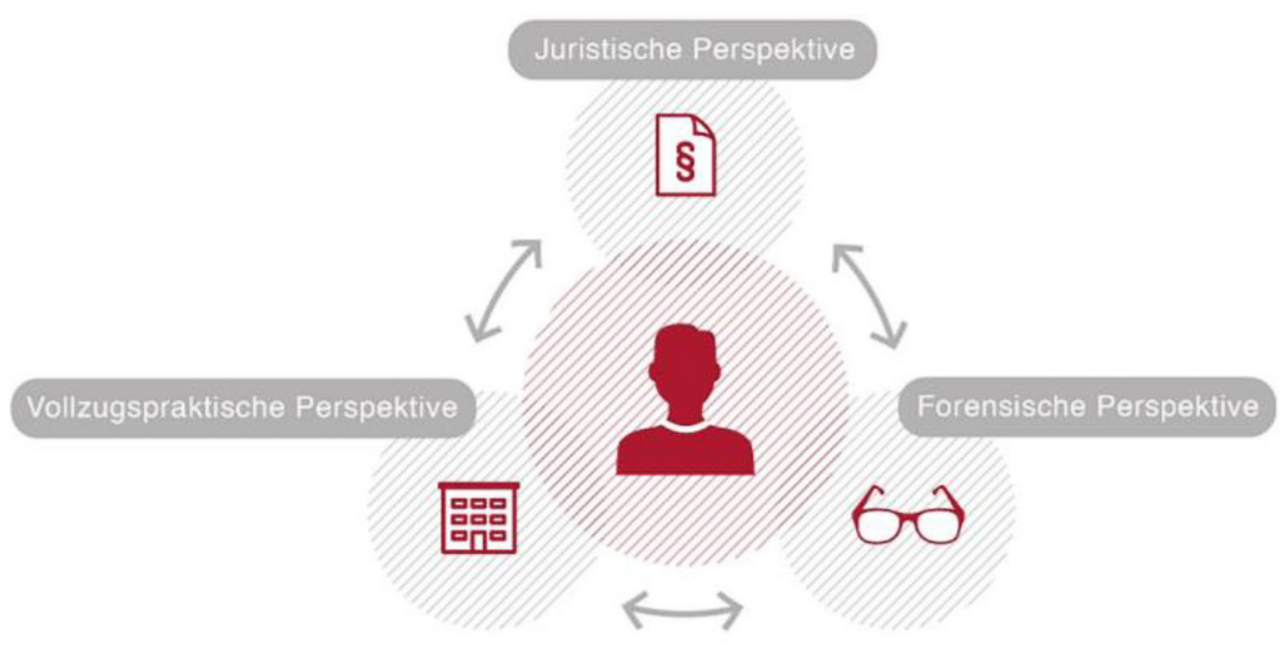

\section{Ziel}

Der ROS wurde bereits in diversen Evaluationen in Bezug auf verschiedene Aspekte betrachtet. Die Prozessevaluation (Ruflin et al. 2013), die parallel zur Testphase von ROS durchgeführt wurde, konnte zeigen, dass ROS diverse positive Auswirkungen auf den Sanktionenvollzug hat. Diese sind u.a. erhöhte Sensibilisierung für Rückfallrisiken, Etablierung einer einheitlichen Terminologie, erhöhte Nachvollziehbarkeit des Fallverlaufs durch standardisierte Dokumentation und Verstärkung der Zusammenarbeit an den Schnittstellen zwischen verschiedenen Arbeitspartnern. Nach der Testphase von ROS wurde außerdem eine Ergebnisevaluation durchgeführt (Schwarzenegger et al.
2013), die diverse Instrumente im ROS-Prozess untersucht hat. Dabei konnten deren Reliabilität und Validität bestätigt werden. Des Weiteren wurde insbesondere das FaST mehrfach evaluiert und optimiert (Schwarzenegger et al. 2013; Loewe-Baur 2017; Treuthardt et al. 2018, Treuthardt und Kröger 2019). Hier konnten ebenfalls die Validität und Reliabilität des FaST bestätigt werden. Offen blieb nun noch, wie sich Veränderungen im Prozessschritt Verlauf standardisiert erfassen lassen. Das ist erforderlich, um auch dynamischen Entwicklungen adäquat Rechnung tragen zu können. Im Folgenden wird nun ein Konzept erarbeitet, anhand dessen $\mathrm{zu}$ einem späteren Zeitpunkt eine Veränderungsmessung der umweltbezogenen Aspekte und personenbezogenen Denk- und Verhaltensmuster der Straf- 
täter und Straftäterinnen durchgeführt werden kann. Diese Veränderungsmessung soll einen weiteren Schritt zur Evaluation der Wirksamkeit des ROS darstellen sowie neue Elemente einführen, die die Abbildung von Veränderungen transparenter und verständlicher machen sollen.

\section{Methode}

Die Veränderungsmessung erfolgt anhand folgender Parameter: Veränderungspotenzial, delinquenzbezogene Prozessvariablen, „stages of change“, umweltbezogene Verhaltensmessung und Ausprägung des umwelt- und personenbezogenen Problemprofils. Um eine bessere Übersicht $\mathrm{zu}$ gewährleisten, werden die Messzeitpunkte im ROS-Prozess nachfolgend in Abb. 3 vorweggenommen.

\section{Personenbezogene Veränderungsmessung}

Personenbezogene Veränderungen beziehen sich - wie der Name sagt - auf Denk- und Verhaltensmuster, die in der Person verankert sind (Treuthardt 2017). Dies können beispielsweise unzureichende Konflikt- oder Stressbewältigungsstrategien, Suchterkrankungen, Schizophrenie, Persönlichkeitsstörungen oder antisoziale Einstellungen sein. Eine nachhaltige Veränderung in diesen Bereichen kann in der Regel nur anhand intensiver Bearbeitung mithilfe von Interventionen erfolgen. Dementsprechend erstreckt sich eine Veränderung über lange Zeiträume.

Veränderungspotenzial Das Veränderungspotenzial wird im ROS-Prozessschritt Abklärung eingeschätzt, also zu Beginn der Sanktion. Es setzt sich zusammen aus der Veränderungsfähigkeit und der Veränderungsmotivation.

Veränderungsfähigkeit Die Veränderungsfähigkeit fragt nach grundlegenden, eher statischen Aspekten. Sie beruht auf den Variablen ausgeprägte dissoziale/impulsive Züge, Vorstrafen und abgebrochene frühere psychologische oder psychiatrische Behandlung. Ausgewählt wurden diese Variablen aufgrund ihres wiederholt bestätigten Zusammenhangs zur Rückfälligkeit (Fries et al. 2011; Glaubitz et al. 2017; Hanson und Bussière 1998; Olver et al. 2011). Hierbei wurden übereinstimmend moderate Effekte zwischen ausgeprägten dissozialen/impulsiven Zügen und Rückfälligkeit gefunden. $\mathrm{Zu}$ ausgeprägten dissozialen/impulsiven Zügen gehören neben einer diagnostizierten dissozialen Persönlichkeitsstörung auch Aspekte wie ein hoher PCLR-Score, deutlich prokriminelle Einstellungen oder außergewöhnliche Feindseligkeit sowie allgemeine Impulsivität in mehreren Lebensbereichen. Zwischen Vorstrafen und Rückfälligkeit wurden ebenfalls übereinstimmend moderate Effekte gefunden; ebenso wie zwischen abgebrochener früherer psychiatrischer Behandlung und Rückfälligkeit. Weitere in den Studien erwähnte Faktoren wurden nicht miteinbezogen, weil sie entweder nicht reliabel erhoben werden konnten (wie Jugendkriminalität oder Bildungsniveau) oder übereinstimmend keine Signifikanz gefunden wurde (wie Intelligenz, neurokognitive Einschränkungen oder Beziehungsstatus). Die 3 Variablen der Veränderungsfähigkeit werden dichotom erfasst, nach vorhanden oder nichtvorhanden.

Veränderungsmotivation Die Veränderungsmotivation deckt grundsätzlich 3 Bereiche ab: Wollen, Wissen und Können (Abb. 4). Die Erfassung erfolgt anhand einer 4-stufigen Likert-Skala (- kaum vorhanden, - etwas vorhanden, + moderat vorhanden, ++ deutlich vorhanden). Im Bereich Wollen wird nach dem risikorelevanten Problembewusstsein, der Verantwortungsübernahme für die getroffenen risikorelevanten Entscheidungen, der Legalbewährungsabsicht und der risikorelevanten Veränderungsbereitschaft gefragt. Der Bereich Wissen umfasst Kenntnisse einerseits zum Problemprofil und andererseits zu deliktpräventiven Strategien. Im Bereich Können wird dann nach der bisherigen Umsetzung der deliktpräventiven Strategien gefragt.

Abschließend erfolgt eine Gesamtbewertung des Veränderungspotenzials ebenfalls auf einer 4-stufigen Likert-
Abb. 3 Messzeitpunkte im Prozess des Risikoorientierten Sanktionenvollzugs

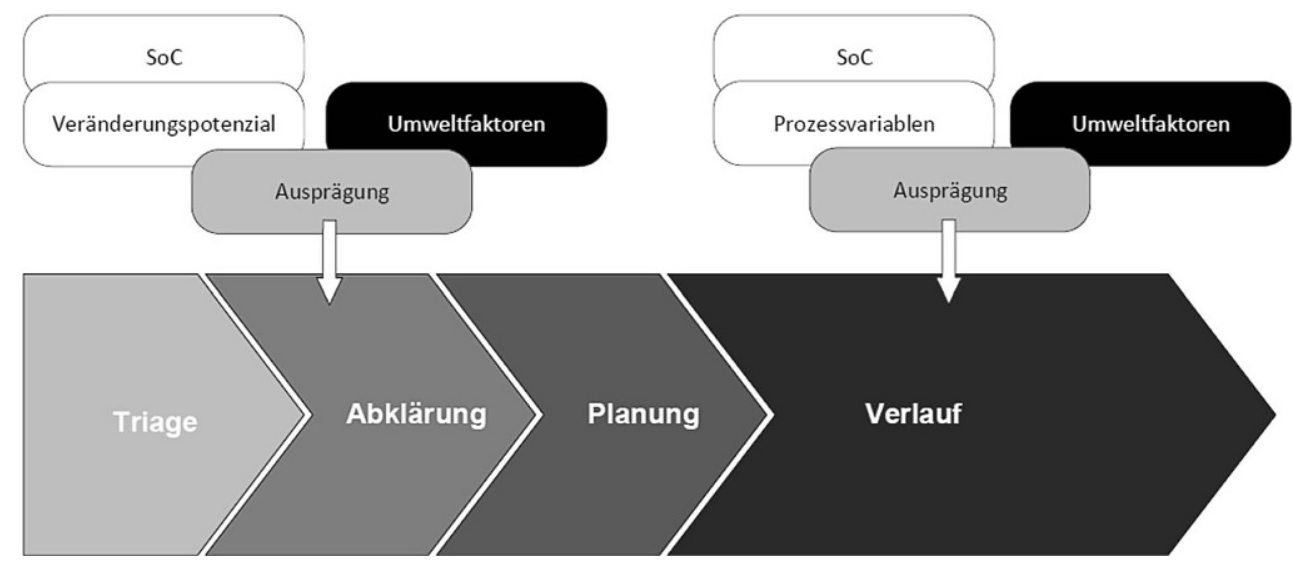




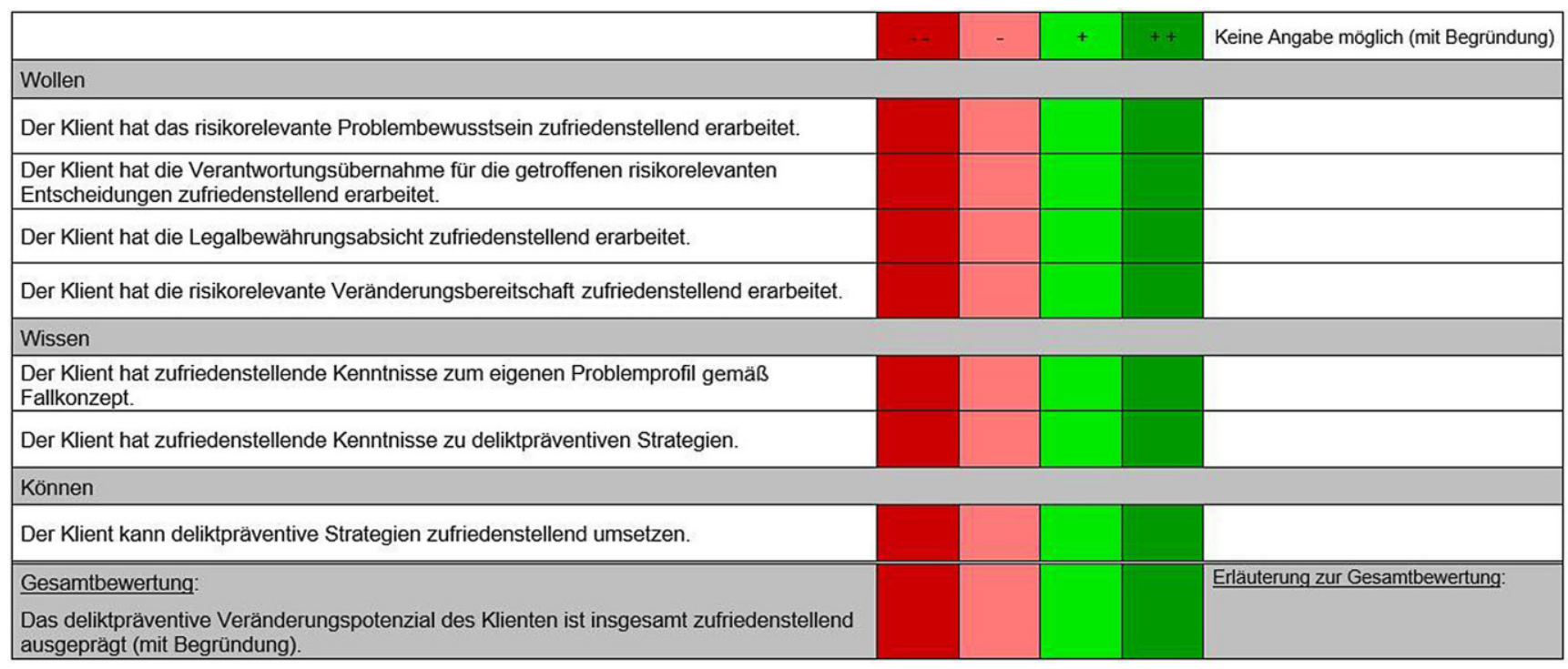

Abb. 4 Veränderungsmotivation

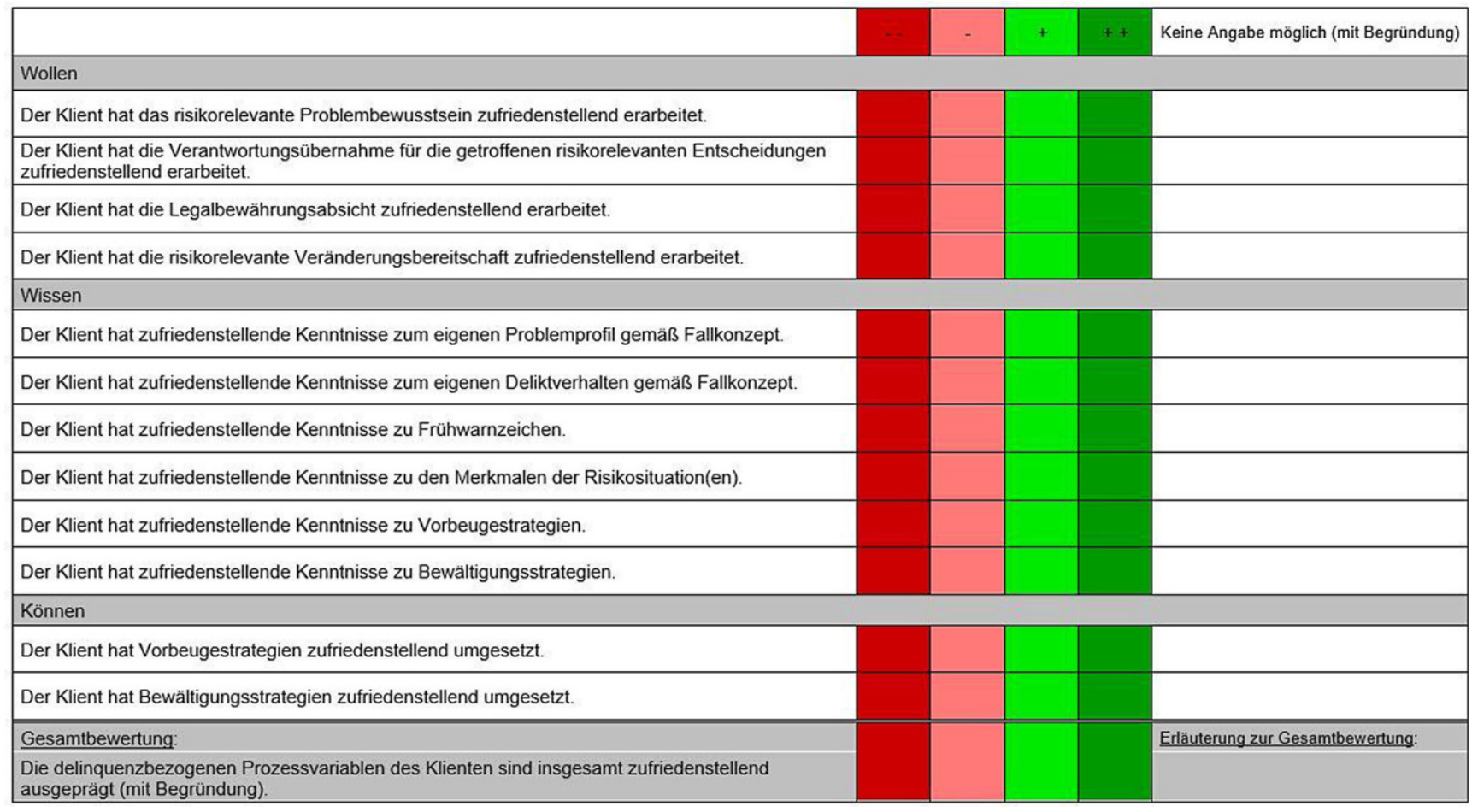

Abb. 5 Delinquenzbezogene Prozessvariablen

Skala (- kaum vorhanden, - etwas vorhanden, + moderat vorhanden, ++ deutlich vorhanden). Die Gesamtbewertung erfolgt nicht anhand einer rein statistischen Auswertung der Bewertungen der Bereiche Wollen, Wissen und Können. Vielmehr soll, unter Berücksichtigung der Bewertungen, eine übergeordnete Einschätzung getroffen werden, die u. U. auch aus den Unterlagen ersichtliche weitere Einflüsse beachtet (beispielsweise früheres Verhalten im Vollzug, mögliche Manipulationstendenzen, früheres Verhalten nach
Entlassung). Die Gesamtbewertung soll immer ausreichend begründet werden, um Rückschlüsse auf die Ergebnisfindung ziehen zu können.

Delinquenzbezogene Prozessvariablen Die delinquenzbezogenen Prozessvariablen lehnen sich an die Veränderungsmotivation an und erweitern diese (Abb. 5). Wie der Name sagt, werden die Prozessvariablen - im Gegensatz zur Veränderungsmotivation - im Verlauf eingeschätzt. Ebenso 
wie die Veränderungsmotivation beziehen sie sich auf die Bereiche Wollen, Wissen und Können und werden auf einer 4-stufigen Likert-Skala (- kaum vorhanden, - etwas vorhanden, + moderat vorhanden, ++ deutlich vorhanden) eingeschätzt. Ebenfalls erfolgt hier eine Gesamtbewertung, die zwischen kaum vorhanden (-), etwas vorhanden $(-)$, moderat vorhanden $(+)$ und deutlich vorhanden $(++)$ unterscheidet. Die Prozessvariablen grenzen sich, neben dem späteren Zeitpunkt der Einschätzung insofern von der Veränderungsmotivation ab, als dass eine deutlich differenzierte Einschätzung der Ebene Wissen durchgeführt wird. Neben den Kenntnissen zum Problemprofil wird nun auch nach Kenntnissen zum Deliktverhalten, zu Frühwarnzeichen und zu Merkmalen der Risikosituation(en) gefragt. Außerdem werden deliktpräventive Strategien in Vorbeugeund Bewältigungsstrategien aufgeteilt. Diese Unterteilung der deliktpräventiven Strategien wird auch bei der Ebene Können vorgenommen.

Stages of change Die Stages of change (SoC, Stufen der Verhaltensänderung) beruhen auf dem Transtheoretischen Modell von Prochaska und DiClemente $(1984,1986)$. Ursprünglich entwickelt zum Verständnis von Verhaltensänderungen bei Rauchern (Prochaska und DiClemente 1984), werden die SoC heute auf eine Bandbreite von problematischen Verhaltensmustern angewendet, wie körperliche Inaktivität und ungesunde Ernährung (Lippke et al. 2012), Substanzabhängigkeit (Prochaska et al. 1992) oder bei der Behandlung einer Vielzahl psychischer Krankheiten (für einen Überblick: Romano und Peters 2015).

Mittlerweile werden die SoC auch in verschiedenen forensischen Bereichen angewendet und erforscht, beispielsweise bei Intimpartner- und häuslicher Gewalt (Eckhardt et al. 2008), Straftätern mit psychischen Störungen (McMurran et al. 1998) oder Sexualstraftätern (Tierney und McCabe 2004).

Insbesondere in Bezug auf die erfolgreiche Feststellung von Behandlungsmotivation in Verbindung mit den SoC (Anstiss et al. 2011; Austin et al. 2011) zeigen sich weitgehend konsistente Befunde im forensischen Bereich. Ebenso scheinen Interventionen, die sich an der entsprechenden Stufe der SoC orientieren, gute Erfolge zu erzielen (Devereux 2009; Alexander und Morris 2008). Dies deckt sich mit den Befunden des RNR-Prinzips, nach denen eine erfolgreiche Behandlung der Person individuell angemessen sein muss (für einen Überblick über das RNR-Prinzip: Polaschek 2012). Insofern bietet sich eine Erweiterung des RNR-Prinzips um die $\mathrm{SoC}$ an, um Veränderungen in risikorelevanten Denk- und Verhaltensmustern individuell abbilden zu können.

Nach Prochaska et al. (1992) gibt es 5 Stufen der Verhaltensänderung. Entgegen dem Namen ist das SoC-Modell kein klassisches Stufenmodell, da für gewöhnlich kein lineares Durchschreiten der Stufen möglich ist. Vielmehr kann eine Person mehrmals eine oder mehrere Stufen zurückfallen oder überspringen (Yong et al. 2015). Trotzdem wird auch im Folgenden von Stufen der Veränderung gesprochen, die allerdings nicht die Kriterien für Stufen im Sinne der wissenschaftlichen Definition erfüllen ${ }^{1}$.

Grundsätzlich sollten die SoC für jedes problematische Verhaltensmuster isoliert betrachtet werden. Weist eine Person beispielsweise das Problemverhalten Rauchen und das Problemverhalten körperliche Inaktivität auf, ist nicht davon auszugehen, dass die Verhaltensweisen zusammengehören. Dementsprechend würde hier keine übergeordnete SoC zugeordnet werden, sondern jeweils eine für Rauchen und eine für körperliche Inaktivität.

Im Folgenden werden die Stufen entsprechend der deutschen Übersetzung der Violence Risk Scale nach HaubnerMacLean et al. (2013) ins Deutsche übersetzt. In Anlehnung an die Faktorenanalyse, die der Erstellung des gängigen Selbstbeurteilungsfragebogens URICA zur Messung der SoC zugrunde liegt (DiClemente und Hughes 1990), wird die dritte Stufe - Vorbereitung - nicht berücksichtigt. Da der URICA in der Originalversion oder in modifizierter Form bei fast allen Messungen der SoC verwendet wird, ist es auch in der Literatur sehr weit verbreitet, lediglich 4 Stufen zu berücksichtigen.

Absichtslosigkeit Es liegt entweder keine (ausreichende) Problemwahrnehmung vor, oder keine Absicht zur Verhaltensänderung. Personen auf dieser Stufe zeigen oftmals Externalisierungstendenzen.

Absichtsbildung Ein Problembewusstsein ist vorhanden. Es werden ernsthafte Überlegungen angestellt, wie man das Verhalten und die Umwelt ändern könnte, ohne konkrete, offensichtliche Schritte unternommen zu haben. Im Optimalfall wird konkret visualisiert, welche Schritte zur Verhaltensänderung vorgenommen werden müssen.

Handlung Es werden aktive Schritte unternommen, das Verhalten und die Umwelt zu ändern, um das vorliegende Problem zu lösen. Die Anstrengungen zur Verhaltensänderung sind offensichtlich und konkret. Die Veränderungen sind instabil. Die bloße Teilnahme an einer Therapie legitimiert keine Einstufung auf die Handlungsstufe.

Aufrechterhaltung Ein nachhaltiges Veränderungsstreben kann beobachtet werden. Dafür muss ein realistisches Bild über das Selbst, das Risiko und die eigenen Ressourcen vorhanden sein. Unter Umständen wurde eine deutliche

\footnotetext{
${ }^{1}$ Stufen im wissenschaftlichen Sinne müssen klar voneinander abtrennbar sein und werden linear durchlaufen ohne Zurückfallen auf vorherige Stufen oder Überspringen einer Stufe.
} 
Tab. 1 Beispielhafte Aussagen für jede Stage of change

\begin{tabular}{llll}
\hline Absichtslosigkeit & Absichtsbildung & Handlung & Aufrechterhaltung \\
\hline „Ich habe kein Problem.“ & „Ich habe ein Prob- & „Ich arbeite aktiv daran mich zu & „Ich möchte meine Änderung auf- \\
„Ich habe nichts falsch gemacht.“ & lem und sollte daran & ändern.“ & rechterhalten.“ \\
„Wenn der andere nichts gemacht & arbeiten.“ & „Ich kann erste Veränderungen & „Ich kenne Strategien, um auch nach \\
hätte, wäre auch nichts passiert.“ & „Ich möchte nicht mehr & an mir feststellen.“ & meiner Entlassung mit meinem Prob- \\
„Vielleicht habe ich ein Problem, aber & so sein.“ & „Ich bin dabei, mich von & lem zurechtzukommen.“ \\
ich kann eh' nichts daran ändern.“ & „Ich möchte eine The- & schlechten Einflüssen aus mei- & „Ich kann mich auch in Risikositua- \\
& rapie machen.“ & nem Umfeld loszusagen.“ & tionen kontrollieren.“ \\
\hline
\end{tabular}

Distanzierung von schlechten Umwelteinflüssen vorgenommen und aufrechterhalten. Ein Rückfall wird aktiv vermieden.

In Tab. 1 sind beispielhafte Aussagen für jede SoC aufgeführt.

Zeitfaktor Bei der Einstufung muss beachtet werden, dass ein Wechsel zwischen Stufen sehr schnell und tagesabhängig erfolgen kann. Für eine Einstufung muss folglich ein relativ stabiler Verbleib über einen längeren Zeitraum hinweg gegeben sein. Das Zeitintervall und die Stabilität der positiven Verhaltensänderung müssen hierbei relativ zu Dauer und Häufigkeit des problematischen Verhaltens gesehen werden (deutsche Übersetzung der VRS (Wong und Gordon 2000) nach Haubner-MacLean et al. 2013). Grundsätzlich werden die SoC sowohl im Prozessschritt Abklärung als auch im Verlauf eingeschätzt.

Besonderheiten bei Straftätern Die Forschung hat gezeigt, dass sich ein großer Teil der Straftäter zu Beginn ihrer Freiheitsstrafe oder vor Beginn einer Intervention entweder auf der Stufe der Absichtslosigkeit oder der Absichtsbildung befindet (Devereux 2009; Polaschek et al. 2010). Insbesondere bei diesen Straftätern ist es wichtig, ein Fortschreiten auf die Handlungsstufe zu fördern, beispielsweise durch „motivational interviewing“ (Anstiss et al. 2011).

Zudem ist es so, dass sich in vielen Fällen die Chancen für Haftlockerungen und eine frühzeitige Entlassung durch die Teilnahme an einer (gerichtlich oder behördlich) angeordneten Intervention erhöhen. Diese Ausgangslage bewirkt, dass viele Straftäter eine starke extrinsische Veränderungsmotivation aufweisen. Bei der Einstufung muss also darauf geachtet werden, nicht nur verbale Aussagen des Straftäters, sondern auch früheres und aktuelles Verhalten zu berücksichtigen, um so Rückschlüsse auf die intrinsische Motivation ziehen zu können.

Des Weiteren sind Eigenschaften zu berücksichtigen, die eine Einstufung erschweren. Beispielhaft sind hierbei Personen mit pädophilen Neigungen zu nennen. Ein pädophiler Straftäter könnte durchaus eine ausgeprägte Absicht zur Verhaltensänderung haben und deliktpräventive Strategien erarbeiten. Im stationären Setting ohne Lockerungen ist es jedoch in der Regel nicht möglich, zu überprüfen, inwiefern die erarbeiteten Strategien auch in Risikosituationen umgesetzt werden können. In diesem Fall sollten Lockerungen und Ausgänge vorsichtig gewährt werden, um den Straftäter schrittweise mit Risikosituationen zu konfrontieren, damit er lernen kann, die Strategien erfolgreich umzusetzen.

Eine weitere Eigenschaft, die die Einstufung erschwert, sind Manipulationstendenzen. Ein Straftäter mit ausgeprägten Manipulationstendenzen könnte beispielsweise versuchen, eine deutliche, andauernde Veränderung vorzutäuschen, tatsächlich aber keinerlei Veränderungsabsicht haben. Auch in diesem Zusammenhang sind also nicht nur verbale Aussagen, sondern auch früheres und aktuelles Verhalten für die Einstufung sehr relevant.

Generell haben Straftäter im geschlossenen Setting nur eingeschränkte Möglichkeiten, neu erarbeitete Einstellungen und Verhaltensweisen der Handlungs- und insbesondere der Aufrechterhaltungsstufe anzuwenden. Die Person muss die Gelegenheit haben, die geänderten Verhaltensweisen insbesondere auch in Risikosituationen zeigen zu können. Für die Feststellung einer erfolgreichen Bewältigung der Stufe der Aufrechterhaltung wird ein Beobachtungszeitraum von ungefähr 5 Jahren nach bedingter Entlassung vorgeschlagen. Dies stützt sich auf die Erkenntnisse aus der Rückfallforschung bei Straftätern, nach denen Rückfälligkeit größtenteils innerhalb der ersten 2 bis 3 Jahre nach der Entlassung vorkommt (Miraglia und Hall 2011; Schalast 2013). So konnten auch Jehle et al. (2016) in einer deutschlandweiten Rückfalluntersuchung zeigen, dass es innerhalb der ersten 3 Jahre in 35,9\% der Fälle zu einem Rückfall kam. Nach 6 Jahren lag die Rückfallrate bei $44,8 \%$ und nach 9 Jahren bei $48,3 \%$. Dementsprechend stieg die Rückfallrate nach 6 Jahren um $8,9 \%$, während nach 9 Jahren lediglich noch ein Anstieg um 3,5\% gefunden werden konnte. Bezugnehmend auf Rückfallfreiheit erläutern Blumstein und Nakamura (2009), dass sich das Risiko einer erneuten Deliktbegehung - je nach Art des Delikts, das zur Inhaftierung geführt hat - nach 5 bis 8 Jahren der Delinquenzwahrscheinlichkeit der Population annähert, die niemals delinquent war. 


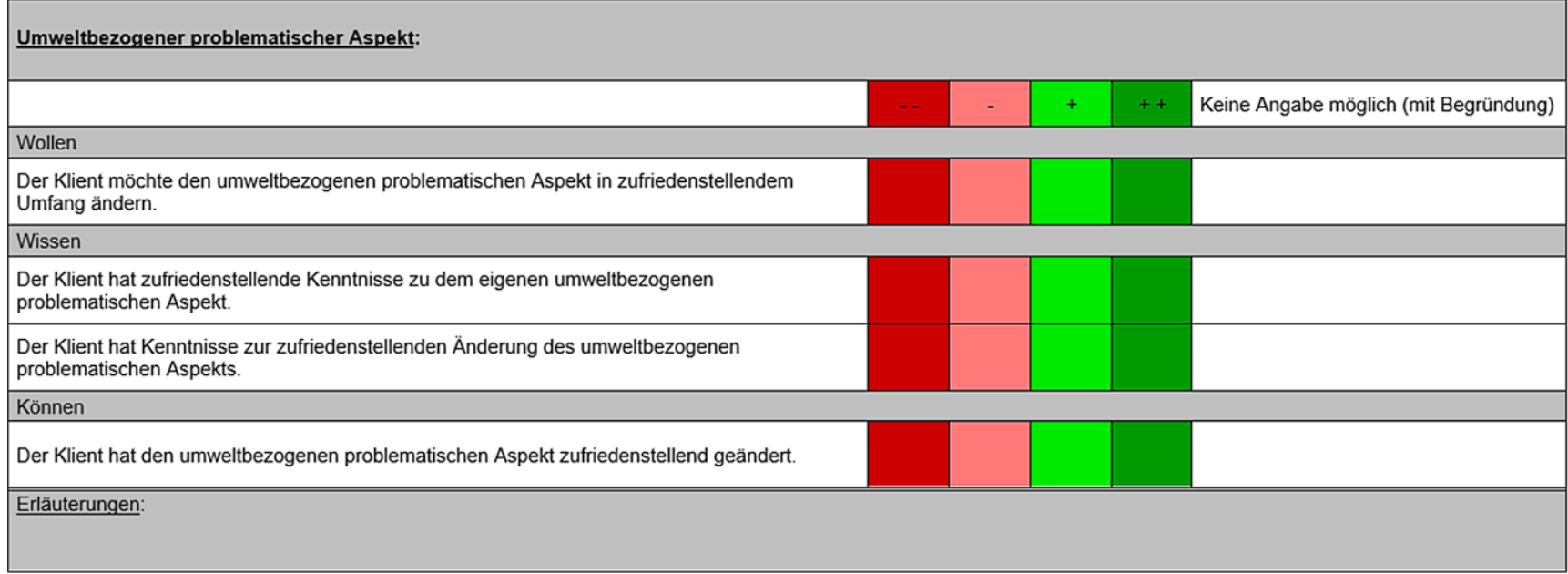

Abb. 6 Umweltbezogene Veränderungsmessung

\section{Umweltbezogene Veränderungsmessung}

Ebenso wie die Veränderungsmotivation und die Prozessvariablen bezieht sich die umweltbezogene Veränderungsmessung auf die Ebenen Wollen, Wissen und Können (Abb. 6). Diese Ebenen werden für jeden umweltbezogenen problematischen Aspekt neu eingeschätzt, auf einer 4-stufigen Likert-Skala (- kaum vorhanden, - etwas vorhanden, + moderat vorhanden, ++ deutlich vorhanden). Im Anschluss werden fallspezifische Erläuterungen eingebracht. Die Einschätzung der umweltbezogenen Veränderung erfolgt während der Prozessschritte Abklärung und Verlauf.

Klassische umweltbezogene Bereiche, in denen Probleme auftauchen, sind Arbeit, Finanzen, Wohnen, Beratung und Betreuung, Freizeit, Partnerschaft und Familie und sonstiges soziales Umfeld. Weitere problematische Bereiche wären beispielsweise Gesundheit oder unklarer Aufenthaltsstatus.

\section{Ausprägung des umwelt- und personenbezogenen Problemprofils}

Die Ausprägung beruht - wie der Name besagt - auf der Ausprägung der risikorelevanten Denk- und Verhaltensmuster. In Anlehnung an die Global-Assessment-ofFunctioning(GAF)-Skala ( $\mathrm{Saß}$ und American Psychiatric Association 1998), die die Beurteilungsgrundlage der Achse V, globale Beurteilung des Funktionsniveaus, nach DSM-IV, darstellt, wird die Ausprägung der Risikoeigenschaften in 5 Kategorien eingeteilt. Die GAF-Skala umfasst 10 Gruppen, die jeweils 10 Codewerte umfassen (1-10, 11-20, 21-30, 31-40, 41-50, 51-60, 61-70, 71-80, 81-90, 91-100). Unter Umbenennung zur Ausprägungsskala werden die Gruppen der ursprünglichen GAF-Skala zusammenfassend umformuliert, um dem forensischen Rahmen zu entsprechen.

Der Umfang der Ausprägung bezieht sich auf die Beeinträchtigung der Persönlichkeit durch spezielle Eigenschaften, die potenziell zu einem Delikt führen. Hierbei wird im Folgenden von ,risikorelevanten Denk- und Verhaltensmustern“ gesprochen. Frei nach der Definition für psychische Störungen nach Wittchen und Hoyer (2011) wird das Ausmaß dieser risikorelevanten Denk- und Verhaltensmuster an ihrer Beeinträchtigung von verhältnismäßigen, psychischen oder biologischen Funktionsfähigkeiten im Alltag geschätzt. Sind risikorelevante Denk- und Verhaltensmuster entsprechend stark ausgeprägt, führen sie also in der Regel zu Einschränkungen und Problemen in verschiedenen zentralen Lebensbereichen (z.B. soziale Kontakte, Beziehungen, Arbeit und Gesundheit). Eine Ausnahme bilden hier insbesondere risikorelevante Denk- und Verhaltensmuster, die sich auf den Bereich der devianten Sexualität beziehen. Diese beeinträchtigen zwar in der Regel die Funktionsfähigkeit in zentralen Lebensbereichen wenig, können jedoch trotzdem stark oder sehr stark ausgeprägt sein. Ein Beispiel hierfür wäre die Pädophilie. Ein pädophiler Mensch kann eine hervorragende Funktionsfähigkeit im Alltag haben, sogar eine eigene Familie gegründet haben, lebt er seine Pädophilie jedoch bei jeder ihm sich bietenden Gelegenheit aus, wäre diese trotzdem deutlich ausgeprägt (Tab. 2).

Sehrgeringe Ausprägung Es liegen keinerlei Einschränkungen der Funktionsfähigkeit oder eine Einschränkung im Normbereich vor. Es liegen keine risikorelevanten Denkund Verhaltensmuster vor. Die Tat begründet sich also ausschließlich auf hochspezifischen situativen und umweltbezogenen Faktoren. 
Tab. 2 Beispiele für Tatpersonen der einzelnen Ausprägungsstufen

\begin{tabular}{|c|c|c|c|c|}
\hline $\begin{array}{l}\text { Sehr geringe Aus- } \\
\text { prägung }\end{array}$ & Geringe Ausprägung & Moderate Ausprägung & Starke Ausprägung & Sehr starke Ausprägung \\
\hline $\begin{array}{l}\text { Eine Mutter sieht, } \\
\text { wie ihrem Kind } \\
\text { Gewalt angetan } \\
\text { wird. Um dies zu } \\
\text { verhindern, schlägt } \\
\text { sie auf die Tatperson } \\
\text { ein. } \\
\text { Ein Mann wird auf } \\
\text { der Straße über- } \\
\text { fallen. Um sich zu } \\
\text { wehren, schlägt er } \\
\text { der angreifenden } \\
\text { Person heftig ins } \\
\text { Gesicht. }\end{array}$ & $\begin{array}{l}\text { Ein eher tempera- } \\
\text { mentvoller Ehemann } \\
\text { schlägt seiner Frau } \\
\text { ins Gesicht, als er von } \\
\text { ihrer Affäre erfährt. } \\
\text { Eine junge Frau ist } \\
\text { in großer Geldnot. } \\
\text { Als einem Passanten } \\
100 € \text { aus der Tasche } \\
\text { fallen, steckt sie diese } \\
\text { ein, statt den Mann } \\
\text { darauf aufmerksam zu } \\
\text { machen. }\end{array}$ & $\begin{array}{l}\text { Eine eigentlich friedliche, aber } \\
\text { beeinflussbare Frau wird von } \\
\text { ihren Freunden überredet, bei } \\
\text { einem Raub mitzumachen. Sie } \\
\text { gibt an, sich häufig von ihren } \\
\text { Freunden ausgenutzt zu fühlen, } \\
\text { weil sie nicht „Nein“ sagen } \\
\text { kann. } \\
\text { In einer von Eifersucht ge- } \\
\text { prägten Beziehung schlägt ein } \\
\text { Mann seine Freundin. Auch auf } \\
\text { der Arbeit hat er gelegentlich } \\
\text { Probleme wegen seiner aufbrau- } \\
\text { senden Art. }\end{array}$ & $\begin{array}{l}\text { Ein pädophiler Pfad- } \\
\text { finderleiter fasst seine } \\
\text { Pfadfinder ungebühr- } \\
\text { lich an, wenn er sich } \\
\text { nicht beobachtet fühlt. } \\
\text { Aufgrund seiner ausge- } \\
\text { prägten Aggressivität } \\
\text { verliert ein Mann häu- } \\
\text { fig Arbeitsstellen, gerät } \\
\text { ständig in Auseinander- } \\
\text { setzungen und schlägt } \\
\text { seine Frau und Kinder. }\end{array}$ & $\begin{array}{l}\text { Ein Mann lockt junge Mäd- } \\
\text { chen mit Fotoshootings, } \\
\text { erstellt dann von ihnen por- } \\
\text { nografische Aufnahmen und } \\
\text { missbraucht sie. } \\
\text { Aufgrund ihrer langjäh- } \\
\text { rigen Drogenproblematik } \\
\text { kann eine Frau nicht arbei- } \\
\text { ten, hat hohe Schulden und } \\
\text { verbringt ihre Zeit mit ande- } \\
\text { ren Abhängigen. Um ihren } \\
\text { Konsum zu finanzieren, be- } \\
\text { geht sie häufig Diebstähle. }\end{array}$ \\
\hline
\end{tabular}

Geringe Ausprägung Es liegen vorübergehende, minimale Einschränkungen der Funktionsfähigkeit vor. Die kurzfristigen Auswirkungen von risikorelevanten Denk- und Verhaltensmustern treten hin und wieder bei spezifischen Umständen auf.

Moderate Ausprägung Es liegen regelmäßige minimale Einschränkungen oder dauerhafte geringe Einschränkungen der Funktionsfähigkeit vor. Die risikorelevanten Denk- und Verhaltensmuster treten bei spezifischen Umständen sehr wahrscheinlich auf.

Starke Ausprägung Es liegen dauerhafte Einschränkungen der Funktionsfähigkeit vor, die mitunter stark ausgeprägt sind. Die risikorelevanten Denk- und Verhaltensmuster wirken sich bei Umständen aus, die wahrscheinlich und häufig auftreten.

Sehr starke Ausprägung Es liegen dauerhafte, starke Einschränkungen der Funktionsfähigkeit vor, die in der Regel mit Selbst- oder Fremdgefährdung oder Realitätsverlust einhergehen. Die risikorelevanten Denk- und Verhaltensmuster sind alltagsrelevant und unabhängig von den Umständen. Mitunter werden risikorelevante Situationen aktiv geschaffen.

In Tab. 2 sind beispielhaft Tatsituationen und -personen der einzelnen Ausprägungsstufen aufgeführt.

Im Zusammenhang mit der Ausprägungsskala steht die Unterscheidung zwischen Situationstätern und Persönlichkeitstätern (Urbaniok 2016). Beim Situationstäter ergibt sich die Tatmotivation aus einer Tatausgangssituation, weitgehend unabhängig von stabilen Persönlichkeitsmerkmalen. Dies umfasst insbesondere die Stufe sehr geringe Ausprägung und weitgehend auch die Stufe geringe Ausprägung.

Beim Persönlichkeitstäter ergibt sich die Tatmotivation aus der Persönlichkeit des Täters. Es werden mitunter Situationen aufgesucht, die eine Straftat bedingen, oder die- se Situationen werden gezielt geschaffen. Dies umfasst die Stufen starke Ausprägung und sehr starke Ausprägung.

Von einer konkreten Einteilung der Stufe moderate Ausprägung zum Situationstäter oder Persönlichkeitstäter wird hier abgesehen.

Wichtig zu beachten ist, dass die Schwere oder die Häufigkeit der Delikte nicht aus der Ausprägung ableitbar ist. Ebenso ist umgekehrt für die Einschätzung der Ausprägung weder die Qualität noch die Quantität der Delikte entscheidend. Es besteht jedoch gesamthaft ein Zusammenhang.

Die Ausprägung des umwelt- und personenbezogenen Problemprofils wird insbesondere im Prozessschritt Abklärung eingeschätzt. Auch während des Verlaufs sollte die Ausprägung beobachtet werden, allerdings ist eine Änderung der Ausprägung, wie bereits beschrieben, meist nur sehr langfristig möglich.

\section{Diskussion}

\section{Potenzial für die Vollzugspraxis}

Entsprechend dem Ziel des ROS schafft die Veränderungsmessung die Möglichkeit, eine Veränderung und bestenfalls Reduktion des risikorelevanten Problemprofils abzubilden. So können insbesondere vollzugspraktische Entscheidungen wie Lockerungen oder Entscheidungen zur bedingten Entlassung transparenter und verständlicher begründet und kommuniziert werden.

Des Weiteren kann anhand der Veränderungsmessung zumindest ansatzweise eine Brücke zwischen juristischen Rahmenbedingungen, vollzugspraktischen Möglichkeiten und forensischem Veränderungsbedarf geschaffen werden. In den Abklärungen, die nun seit mehreren Jahren gemäß dem Konzept des ROS zur Anwendung kommen, wird nicht selten festgestellt, dass dem individuellen forensischen Veränderungsbedarf nicht entsprochen werden kann, 
da die richterlich angeordnete Sanktion keine Möglichkeit einer forensisch-psychologischen Intervention vorsieht. Dementsprechend ist von forensisch-psychologischer Seite davon auszugehen, dass keine ausreichende Veränderung des risikorelevanten Problemprofils erreicht werden kann. Anhand der vorgestellten Veränderungsmessung kann im Verlauf der Sanktion nachvollziehbar dokumentiert werden, ob tatsächlich keine Verbesserung der risikorelevanten umweltbezogenen Aspekte und personenbezogenen Denk- und Verhaltensmuster stattgefunden hat oder ob diese mitunter ausgeprägter geworden sind. Auf diese Weise könnte bei einer negativen Entwicklung eine solide Argumentationsgrundlage für risikomindernde Therapie- oder Unterstützungsangebote geschaffen werden. In einem anderen Fall werden evtl. vollzugspraktische Interventionsmöglichkeiten genutzt, um beispielsweise gezielt an einer Förderung der Veränderungsmotivation zu arbeiten, wodurch bei der verurteilten Person ein risikosenkender Veränderungsprozess ausgelöst wird. Dieser kann durch die Anwendung der vorgeschlagenen Parameter messbar gemacht werden.

\section{Kritik}

Die wohl größte Herausforderung für die hier konstruierte Veränderungsmessung ist die Interrater-Reliabilität. Zwar müssen alle Einschätzungen begründet getroffen werden, jedoch wird eine vollkommene Übereinstimmung in den Einschätzungen voraussichtlich nicht erreicht werden können. Dementsprechend wird es in der Praxis entscheidend sein, Bewertungen mit den entsprechenden Arbeitspartnern zu besprechen und insbesondere abweichende Einschätzungen eingehend zu betrachten.

Des Weiteren ist eine valide Einschätzung nur möglich, wenn eine möglichst umfassende und diverse Bewertungsgrundlage vorhanden ist. Insbesondere sollten nicht nur Aussagen der einzuschätzenden Person zugrunde gelegt werden, sondern auch fremdanamnestische Quellen und bestenfalls eigene Beobachtungen hinzugezogen werden. Dies ist insofern besonders relevant, als dass bei Straftätern und Straftäterinnen häufig eine erhöhte extrinsische Motivation vorhanden ist. So kann beispielsweise eine freiwillige Teilnahme an einem Antiaggressionsprogramm eine bedingte Entlassung begünstigen. Gleichzeitig kann es jedoch sein, dass die betreffende Person tatsächlich keine intrinsische Änderungsmotivation und -absicht hat. Fällt die externe Verstärkung (in diesem Fall die Aussicht auf eine bedingte Entlassung) weg, verschwinden möglicherweise auch die im besten Fall erlernten Denk- und Verhaltensmuster aus dem Antiaggressionsprogramm.

\section{Fazit und Ausblick}

Die hier konstruierte Veränderungsmessung soll Veränderungen transparenter und nachvollziehbarer machen. Sie stellt eine Erweiterung der bereits vorhandenen standardisierten formalen Berichtsauswertung gemäß dem ROS dar. Gleichzeitig stellt die Interrater-Reliabilität eine Herausforderung dar, die es insbesondere durch intensiven Austausch zu bewältigen gilt. Anhand der vorgestellten Messung soll eine Evaluation durchgeführt werden, die retrospektiv Veränderungen von Personen im ROS betrachtet. So soll u. a. festgestellt werden, inwieweit sich Personen während der Sanktion verändern und inwiefern Veränderungen mit Rückfällen zusammenhängen. In diesem Zusammenhang soll auch die Interrater-Reliabilität der Veränderungsmessung untersucht werden.

Funding Open access funding provided by University of Zurich

Interessenkonflikt D. Treuthardt war u.a. an der Entwicklung und Einführung des ROS beteiligt. Außerdem ist D. Treuthardt Leiter der Bewährungs- und Vollzugsdienste des Kantons Zürich und der ROS-Administration, die die Konzeptverantwortung für ROS trägt. D. Treuthardt gibt an, dass dies keinen Interessenskonflikt darstellt, im Rahmen wissenschaftlicher Transparenz jedoch explizit offenzulegen ist. M. Kröger gibt an, dass kein Interessenskonflikt besteht.

Open Access Dieser Artikel wird unter der Creative Commons Namensnennung 4.0 International Lizenz veröffentlicht, welche die Nutzung, Vervielfältigung, Bearbeitung, Verbreitung und Wiedergabe in jeglichem Medium und Format erlaubt, sofern Sie den/die ursprünglichen Autor(en) und die Quelle ordnungsgemäß nennen, einen Link zur Creative Commons Lizenz beifügen und angeben, ob Änderungen vorgenommen wurden.

Die in diesem Artikel enthaltenen Bilder und sonstiges Drittmaterial unterliegen ebenfalls der genannten Creative Commons Lizenz, sofern sich aus der Abbildungslegende nichts anderes ergibt. Sofern das betreffende Material nicht unter der genannten Creative Commons Lizenz steht und die betreffende Handlung nicht nach gesetzlichen Vorschriften erlaubt ist, ist für die oben aufgeführten Weiterverwendungen des Materials die Einwilligung des jeweiligen Rechteinhabers einzuholen.

Weitere Details zur Lizenz entnehmen Sie bitte der Lizenzinformation auf http://creativecommons.org/licenses/by/4.0/deed.de.

\section{Literatur}

Alexander PC, Morris E (2008) Stages of change in batterers and their response to treatment. Violence Vict 23(4):476-492. https://doi. org/10.1891/0886-6708.23.4.476

Andrews DA, Bonta J (2010) The psychology of criminal conduct. Routledge, London

Anstiss B, Polaschek DL, Wilson M (2011) A brief motivational interviewing intervention with prisoners: when you lead a horse to water, can it drink for itself? Psychol Crime Law 17(8):689-710. https://doi.org/10.1080/10683160903524325

Austin KP, Williams MWM, Kilgour G (2011) The effectiveness of motivational interviewing with offenders: an outcome evaluation. NZ J Psychol 40(1):55-67 
Blumstein A, Nakamura K (2009) "Redemption" in an era of widespread criminal background checks. Criminology 47(2):327-359. https://doi.org/10.1111/j.1745-9125.2009.00155.x

Devereux R (2009) Motivating offenders to attend (and benefit from) rehabilitative programmes: The development of a short motivational programme within New Zealand's Department of Corrections. J N Z Coll Clin Psychol 19(3):6-14

DiClemente CC, Hughes SO (1990) Stages of change profiles in outpatient alcoholism treatment. J Subst Abuse 2(2):217-235. https:// doi.org/10.1016/s0899-3289(05)80057-4

Eckhardt C, Holtzworth-Munroe A, Norlander B, Sibley A, Cahill M (2008) Readiness to change, partner violence subtypes, and treatment outcomes among men in treatment for partner assault. Violence Vict 23(4):446-475. https://doi.org/10.1891/0886-6708.23. 4.446

Fries D, Endrass J, Ridinger M, Urbaniok F, Rossegger A (2011) Indikatoren für den Verlauf einer stationären Behandlung bei Straftätern mit Substanzabhängigkeit. Fortschr Neurol Psychiatr 79(9):404-410. https://doi.org/10.1055/s-0029-1245962

Glaubitz C, Bliesener T, Klatt T (2017) Junge Mehrfach- und Intensivtäter, persistierende Delinquenz und ,callous-unemotional traits“. Forens Psychiatr Psychol Kriminol 11(4):349-354. https://doi. org/10.1007/s11757-017-0442-3

Hanson RK, Bussière MT (1998) Predicting relapse: a meta-analysis of sexual offender recidivism studies. J Consult Clin Psychol 66(2):348-362. https://doi.org/10.1037/0022-006x.66.2.348

Haubner-MacLean T, Heurix I, Eher R (2013) Violence Risk Scale (VRS) (Deutsche Übersetzung)

Jehle JM, Albrecht HJ, Hohmann-Fricke S, Tetal C (2016) Legalbewährung nach strafrechtlichen Sanktionen: Eine bundesweite Rückfalluntersuchung 2010 bis 2013 und 2004 bis 2013. Forum, Godesberg (Bundesministerium der Justiz und für Verbraucherschutz)

Lippke S, Nigg CR, Maddock JE (2012) Health-promoting and healthrisk behaviors: theory-driven analyses of multiple health behavior change in three international samples. IntJ Behav Med 19(1):1-13. https://doi.org/10.1007/s12529-010-9135-4

Loewe-Baur M (2017) Der Risikoorientierte Sanktionenvollzug (ROS). Ergebnisse und Erkenntnisse einer Evaluation. Dissertation, Universität Zürich

McMurran M, Tyler P, Hogue T, Cooper K, Dunseath W, McDaid D (1998) Measuring motivation to change in offenders. Psychol Crime Law 4(1):43-50. https://doi.org/10.1080/10683169808401746

Miraglia R, Hall D (2011) The effect of length of hospitalization on rearrest among insanity plea acquittees. J Am Acad Psychiatry Law 39(4):524-534

Olver ME, Stockdale KC, Wormith JS (2011) A meta-analysis of predictors of offender treatment attrition and its relationship to recidivism. J Consult Clin Psychol 79(1):6-21. https://doi.org/10. 1037/a0022200

Polaschek DL (2012) An appraisal of the risk-need-responsivity (RNR) model of offender rehabilitation and its application in correctional treatment. Leg Criminol Psychol 17(1):1-17. https://doi. org/10.1111/j.2044-8333.2011.02038.x

Polaschek DL, Anstiss B, Wilson M (2010) The assessment of offending-related stage of change in offenders: psychometric validation of the URICA with male prisoners. Psychol Crime Law 16(4):305-325. https://doi.org/10.1080/10683160802698766

Prochaska JO, DiClemente CC (1984) The transtheoretical approach: crossing traditional boundaries of therapy. Dow Jones-Irwin, Homewood, Ill
Prochaska JO, DiClemente CC (1986) Toward a comprehensive model of change. In: Treating addictive behaviors. Springer, Boston, S 3-27

Prochaska JO, DiClemente CC, Norcross JC (1992) In search of how people change: applications to addictive behaviors. Am Psychol 47(9):1102-1114. https://doi.org/10.1037/0003-066x.47.9.110

Romano M, Peters L (2015) Evaluating the mechanisms of change in motivational interviewing in the treatment of mental health problems: a review and meta-analysis. Clin Psychol Rev 38:1-12. https://doi.org/10.1016/j.cpr.2015.02.008

Ruflin R, Miani C, Dvorak A, Jörg R, Schnyder-Walser K (2013) Modellversuch Risikoorientierter Sanktionenvollzug. Schlussbericht Prozessevaluation. https://www.bj.admin.ch/dam/data/bj/ sicherheit/smv/modellversuche/evaluationsberichte/ros-schlussbereval-d.pdf. Zugegriffen: 10. Okt. 2019

$\mathrm{Saß} \mathrm{H}$, American Psychiatric Association (1998) Diagnostisches und statistisches Manual psychischer Störungen DSM-IV. Hogrefe, Göttingen (übersetzt nach der vierten Auflage des Diagnostic and statistical manual of mental disorders der American Psychiatric Association)

Schalast N (2013) Die Dauer der Unterbringung in der Entziehungsanstalt. Forens Psychiatr Psychol Kriminol 7(2):105-113. https:// doi.org/10.1007/s11757-013-0209-4

Schwarzenegger C, Manzoni P, Baur M (2013) Modellversuch Risikoorientierter Sanktionenvollzug. Ergebnisevaluation Schlussbericht. https://www.bj.admin.ch/dam/data/bj/sicherheit/smv/ modellversuche/evaluationsberichte/ros-schlussber-ergebis-d. pdf. Zugegriffen: 10. Okt. 2019

StVollzG NRW, Gesetz zur Regelung des Vollzuges der Freiheitsstrafe in Nordrhein-Westfahlen (Strafvollzugsgesetz Nordrhein-Westfalen) (2015)

Tierney DW, Mccabe MP (2004) The assessment of motivation for behaviour change among sex offenders against children: an investigation of the utility of the stages of change questionnaire. J Sex Aggress 10(2):237-249. https://doi.org/10.1080/ 13552600412331289041

Treuthardt D (2017) Tatpersonen häuslicher Gewalt. Ein delinquenzbezogenes Handlungsmodell für Behörden, Institutionen und Fachpersonen. www.ebg.admin.ch/dam/ebg/de/dokumente/ haeusliche_gewalt/bericht_treuthardt_handlungsmodell_tatperso nen.pdf.download.pdf/14-EBG_2017_Bericht_Treuthardt_Hand lungsmodell_Tatpersonen_d.pdf. Zugegriffen: 11. Okt. 2019

Treuthardt D, Kröger M (2019) Der Risikoorientierte Sanktionenvollzug (ROS) - empirische Überprüfung des Fall-Screening-Tools (FaST). Schweiz Z Kriminol 19(1):76-85

Treuthardt D, Loewe-Baur M, Kröger M (2018) Der Risikoorientierte Sanktionenvollzug (ROS) - aktuelle Entwicklungen. Schweiz Z Kriminol 2018(2):24-32

Treuthardt D, René M, Rohner A (2019) ROS allgemein. https://www. rosnet.ch/de-ch/ros-allgemein. Zugegriffen: 8. Okt. 2019

Urbaniok F (2016) FOTRES-Forensisches Operationalisiertes Therapie-Risiko-Evaluations-System: Diagnostik, Risikobeurteilung und Risikomanagement bei Straftätern. MWV, Berlin

Wittchen HU, Hoyer J (Hrsg) (2011) Klinische Psychologie \& Psychotherapie (Lehrbuch mit Online-Materialien). Springer, Berlin, Heidelberg, New York

Wong S, Gordon A (2000) Violence Risk Scale (VRS)

Yong AD, Williams MWM, Provan H, Clarke D, Sinclair G (2015) How do offenders move through the stages of change? Psychol Crime Law 21(4):375-397. https://doi.org/10.1080/1068316x. 2014.989166 